
«ПРОСТЫЕ АНАЛОГИИ, «СЛОЖНЫЕ АНАЛОГИИ ПРИ ИСПОЛЬЗОВАНИИ ИХ ДЛЯ ОЦЕНКИ НАРУШЕНИЯ МЫШЛЕНИЯ ПРИ ШИЗОФРЕНИИ

\author{
Демянова Л. В. (I_demianova@mail.ru) \\ УО «Гродненский государственный медицинский университет», Гродно, Беларусь
}

Введение. Психометрическая стандартизация диагностических методик является актуальной тенденичией современной медицинской психологии.

Цель. Анализ психометрических свойств методик «Существенные признаки», «Простые аналогии», «Сложные аналогии».

Материал и методы. С помощью методик обследованы 122 пациента с диагнозом шизофрения. Анализ методик включал расчёт трудности заданий, оценку конструктной валидности, показателей надежности и дифференцирующей способности с использованием модели Раша.

Результаты и выводы. Методика «Существеннье признаки» преобразована в 9-типунктовую, в результате чего получено оптимальное сочетание психометрических показателей. На всех этапах анализа методик «Простые аналогии» и «Сложные аналогии» обнаруживается несоответствие ряда показателей психометрическим требованиям, возможность использования этих методик будет определяться результатами оценки согласованности с клиническими данными.

Ключевые слова: шизофрения, нарушения мышления, психометрический анализ, модель Раша

\section{Введение}

В отечественной медицине для исследования мышления принято использовать беседу и патопсихологические методики. Так, «Клинические протоколы оказания медицинской помощи пациентам с психическими и поведенческими расстройствами» (2010) предписывают проводить экспериментально-психологическое обследование для выявления диагностически значимых изменений восприятия, мышления, аффективно-личностных свойств.

Патопсихологические методики для исследования мыслительной деятельности, действительно, чувствительны к нарушениям мышления. Кроме того, их достоинством является простота, пластичность, возможность индивидуального подхода при исследовании [1, 4]. Но направленность на аналитическую качественную оценку экспериментального материала, отсутствие стандартной процедуры исследования затрудняют воспроизводимость результатов и практически невозможно получить сопоставимые данные у разных испытуемых. Современной же тенденцией развития психиатрии является дименсиональный подход, предполагающий объективную количественную оценку исследуемого конструкта. Кроме того, наличия измерительного инструмента требуют современные направления научных исследований мышления при шизофрении (исследование связи нарушений мышления с генетическими факторами, связи нарушений мышления с органическими и функциональными изменениями в головном мозге, влияния фармакотерапии и психотерапии на нарушения мышления и т.д.) $[5,8,10,11,12]$.

Для создания и стандартизации психодиагностических инструментов актуально использование современной теории тестов, в частности ее разновидности - модели Раша $[1,2,3,6,7]$. Модель Раша позволяет конструировать равноинтервальные шкалы и оценивать психометри- ческие свойства диагностических методик на небольшой выборке и получать показатели, не зависящие от выборки. Это обусловлено тем, что модель Раша является вероятностной моделью. Применение модели Раша для стандартизации психодиагностических методик способствует:

- более экономичному применению теста (учитывая время и затраты);

- лучшему теоретическому обоснованию измерения и измеримости рассматриваемого конструкта;

- валидизации одномерных конструктов и выбора пунктов на основе объективных методологических критериев;

- более адекватной интерпретации ответов на тестовые задания;

может внести ощутимый вклад в улучшение качества психиатрических данных [9].

Цель исследования - анализ психометрических свойств методик «Существенные признаки», «Простые аналогии», «Сложные аналогии» при использовании их для оценки выраженности нарушения мышления при шизофрении, преобразование методик до вариантов, обладающих оптимальным сочетанием психометрических показателей.

\section{Материал и методы}

В исследовании принимали участие пациенты женского и мужского отделения УЗ ГОКЦ «Психиатрия-Наркология» $(\mathrm{n}=122)$ с установленным по результатам клинико-психопатологической диагностики в рамках исследовательских диагностических критериев МКБ-10 диагнозом шизофрения. Группа исследования включала 69 женщин и 53 мужчины, возраст пациентов - от 18 до 60 лет, распределение по формам шизофрении следующее: параноидная - 79 человек, простая - 39 человек, кататоническая - 3 человека, гебефренная - 1 человек.

Пациенты были обследованы клинически и патопсихологически с помощью батареи ото- 
бранных нами семи методик («Сходство», «Исключение лишнего», «Существенные признаки», «Простые аналогии», «Сложные аналогии», «Пословицы», «Направленный ассоциативный эксперимент»). Критериями выбора методик были: направленность на исследование мышления; структура методики в виде заданий, каждое из которых можно оценить отдельно; возможность количественной оценки результата выполнения задания.

Методики «Существенные признаки», «Простые аналогии», «Сложные аналогии» взяты нами из книги «Экспериментальные методики патопсихологии» С. Я. Рубинштейн [4].

Методика «Существенные признаки» используется для исследования логичности суждений пациента, способности дифференцировать существенные признаки предметов или явлений и несущественные, второстепенные.

Выполнение заданий методики «Простые аналогии» требует понимания логических связей и отношений между понятиями.

Методика «Сложные аналогии» направлена на выявление того, в какой мере обследуемому доступно понимание сложных логических отношений и выделение абстрактных связей.

В нашем исследовании после предъявления инструкции пациент самостоятельно отмечал правильные, на его взгляд, варианты ответа на задания методики.

Чтобы оценить выраженность нарушения мышления, нами предложена в методике «Существенные признаки» оценка ответов по системе 0-1-2: 0 - правильно выбраны два слова, имеющие наибольшее отношение к слову за скобками; 1 - правильно выбрано одно слово, второе является необязательной характеристикой слова за скобками; 2 - оба выбранных слова не являются существенными признаками слова за скобками, либо пациент подчеркнул меньше или больше, чем два слова.

Для методик «Простые аналогии» и «Сложные аналогии» предложена оценка ответов 0-1, т.е. правильный и неправильный ответ (соответственно).

По ответам всех испытуемых на все задания (пункты) были созданы матрицы данных каждой из методик, которые с помощью модели Раша преобразованы в матрицы вероятностей. На основании матриц вероятностей произведены расчёт трудности пунктов, оценка конструктной валидности пунктов, определение показателей надежности и дискриминативности и факторный анализ остатков, позволяющий определить одномерность или неодномерность методики.

\section{Результаты и обсуждение}

Меры трудностей диагностических заданий методик «Существенные признаки» и «Сложные аналогии», рассчитанные с помощью модели Раша, оказались соответствующими средней степени выраженности исследуемого конструкта (от -2 до 2 логитов). Расчет трудности пунктов методики «Простые аналогии» показал, что пункт № 1 обладает чрезмерной трудностью
(3,2 логита), т.е. на него редко дается ключевой ответ. В нашем случае это ответ, отражающий нарушение мышления, соответствующий оценке 1, т.е. на это задание большинство обследуемых отвечают правильно. Поэтому данный пункт был исключен из дальнейшей обработки информации.

Далее оценивалась конструктная валидность пунктов на основе значений индексов качества пунктов (UMS - Weighted Mean Square и WMS - Unweighted Mean Square). Пункты, имевшие чрезмерно высокие значения индексов UMS и WMS, исключались из дальнейшего анализа как нарушающие конструктную валидность шкалы, осуществлялось повторное моделирование.

Значения индексов качества всех оставшихся пунктов попали в диапазон приемлемых значений для методики «Существенные признаки» на пятой итерации и в методике осталось 16 пунктов.

Факторный анализ нормализованных остатков от разницы между наблюдаемыми и ожидаемыми оценками ответов на пункты методики «Существенные признаки» показал неодномерность как исходного варианта, так и 16-пунктового, т.е. на результат оказывают влияние не только нарушение мышления, но какие-то другие факторы. При анализе методики, состоящей из 20-ти пунктов, три фактора остатков оказались выше 2, что говорит о их существенном влиянии на результат (более $36 \%$ кумулятивной дисперсии). В 16-пунктовой методике один фактор остатков превысил 2 и составил почти 2,5 (15,4\% кумулятивной дисперсии) (рис. 1).
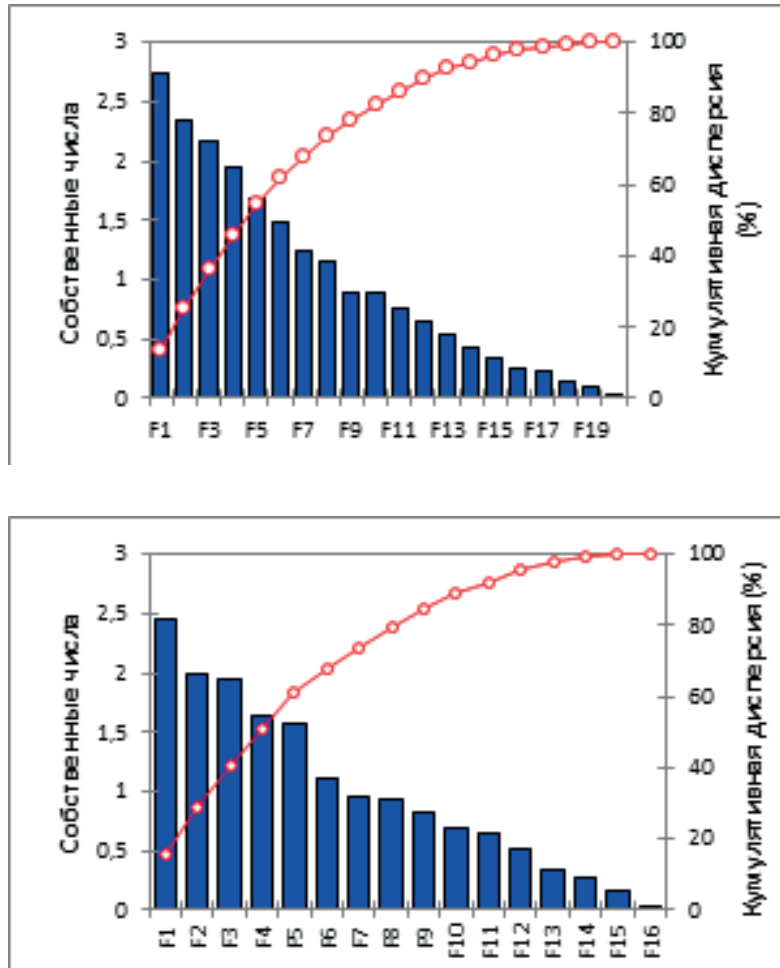

Рисунок 1. - Диаграмма собственных чисел и график россыпи факторов матрицы остатков исходной и 16-пунктовой методики «Существенные признаки» 
Анализ факторных нагрузок пунктов, составивших F1 в 16-пунктовой методике, показал, что этот фактор формируется преимущественно за счет трех пунктов: №№ 9, 10, 12 (факторная нагрузка больше 0,4$)$. Мы исключили их из методики и рассчитали психометрические показатели по оставшимся 13-ти заданиям.

Расчет индексов качества выявил, что конструктная валидность 13-пунктовой методики нарушена. В связи с этим появилась необходимость вновь произвести серию итераций, удаляя пункты, имеющие высокие значения индексов WMS и/или UMS. Было выполнено четыре итерации, после которых осталось 9 пунктов, характеризующихся удовлетворительными значениями индексов качества.

Факторный анализ нормализованных остатков от разницы между наблюдаемыми и ожидаемыми оценками ответов на пункты методики показал одномерность 9-пунктового варианта методики «Существенные признаки» (рис. 2).

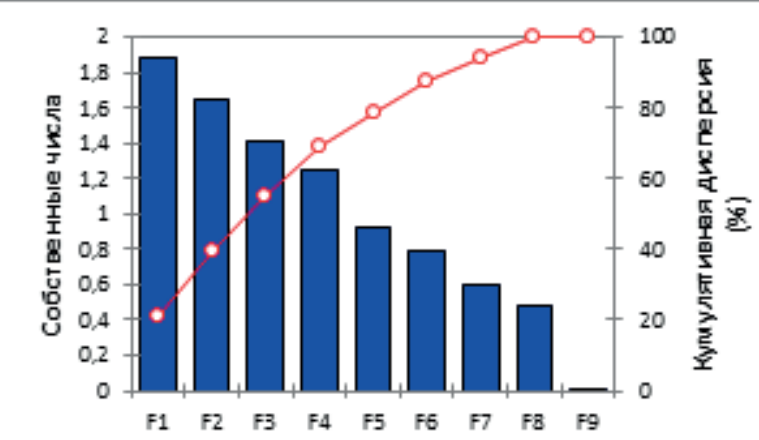

Рисунок 2. - Диаграмма собственных чисел и график россыпи факторов матрицы остатков 9-пунктовой методики "Существенные признаки»

Отдельно для каждой итерации рассчитывались показатели надежности и дискриминативности (количество слоев). Высокий изначально Индекс надежности $(0,88)$ при уменьшении количества пунктов несколько уменьшился, но остался высоким (для 9-пунктовой методики составил 0,866). Количество слоев также изменилось несущественно (от 3,9 для исходной методики до 3,7 для 9-пунктовой). Т.е. методика может дифференцировать три уровня выраженности нарушения мышления, что является показателем достаточных дискриминативных свойств.

Последним этапом психометрического анализа было построение карты, отражающей соотношение мер трудностей ответных категорий пунктов методики «Существенные признаки» и мер выраженности нарушения мышления в группе испытуемых. На рис. 3 представлена карта соотношения этих мер.

Визуальный анализ распределения мер трудности показывает, что ответные категории пунктов локализуются в диапазоне от $-5,0$ до 5 логитов и в этом же диапазоне распределены оценки подавляющего большинства испытуемых. Таким

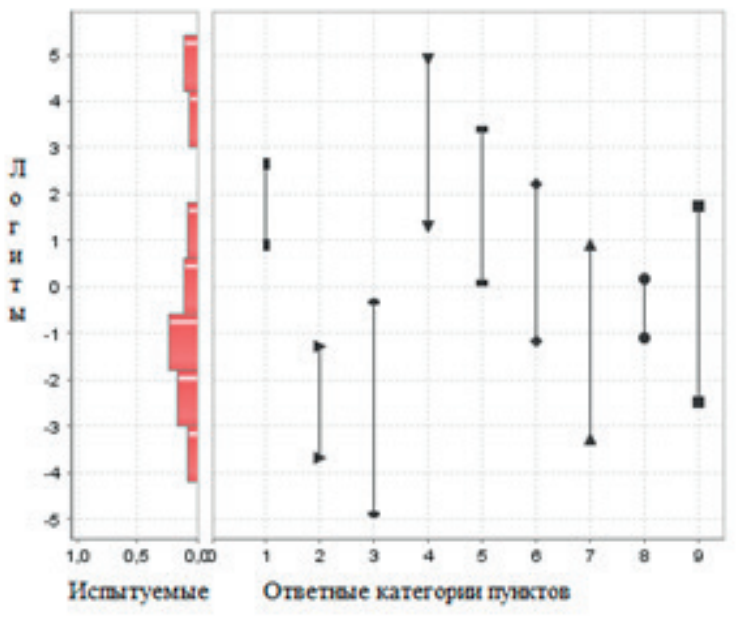

Рисунок 3. - Карта распределения мер выраженности нарушения мышления у испытуемых и трудностей ответных категорий пунктов 9-пунктовой методики "Существенные признаки"

образом, 9-пунктовая методика «Существенные признаки» имеет сбалансированную метрическую структуру, характеризующуюся адекватным соответствием распределения оцениваемых уровней нарушения мышления и диагностических трудностей ответных категорий пунктов.

Для получения удовлетворительной конструктной валидности пунктов методики «Простые аналогии» было произведено 12 итераций по вычислению индексов WMS и UMS, после которых осталось 10 пунктов, имеющих значения обоих индексов в пределах 1,3.

Но при этом Индекс надежности и количество слоев снижались с каждой итерацией. Уже на третьей итерации показатель количества слоев упал ниже 3 (при изначальном - 3,95). Способность методики дифференцировать менее трех уровней выраженности свойства существенно снижает прикладное значение ее использования. Индекс надежности снизился с высокого на первой итерации $(0,88)$ до удовлетворительного на последней $(0,77)$.

Факторный анализ нормализованных остатков от разницы между наблюдаемыми и ожидаемыми оценками ответов на пункты методики «Простые аналогии» показал неодномерность всех вариантов методики. Однако, если в исходной методике четыре фактора превысили 2 и составили 54\% кумулятивной дисперсии (т.е. более половины результата определяется не выраженностью нарушения мышления, а какими-либо другими факторами), то в окончательном 10-пунктовом варианте выделился только один фактор остатков, превысивший 2.

Кроме того, анализ соотношения мер трудностей пунктов методики «Простые аналогии» и мер выраженности нарушения мышления в группе испытуемых показал, что меры трудностей пунктов охватывают около 30\% мер выраженности нарушения мышления в группе испы- 
туемых. А к последней итерации еще меньше, т.к. образовался участок выраженности нарушения мышления, в который не попал никто из пациентов (рис. 4.).
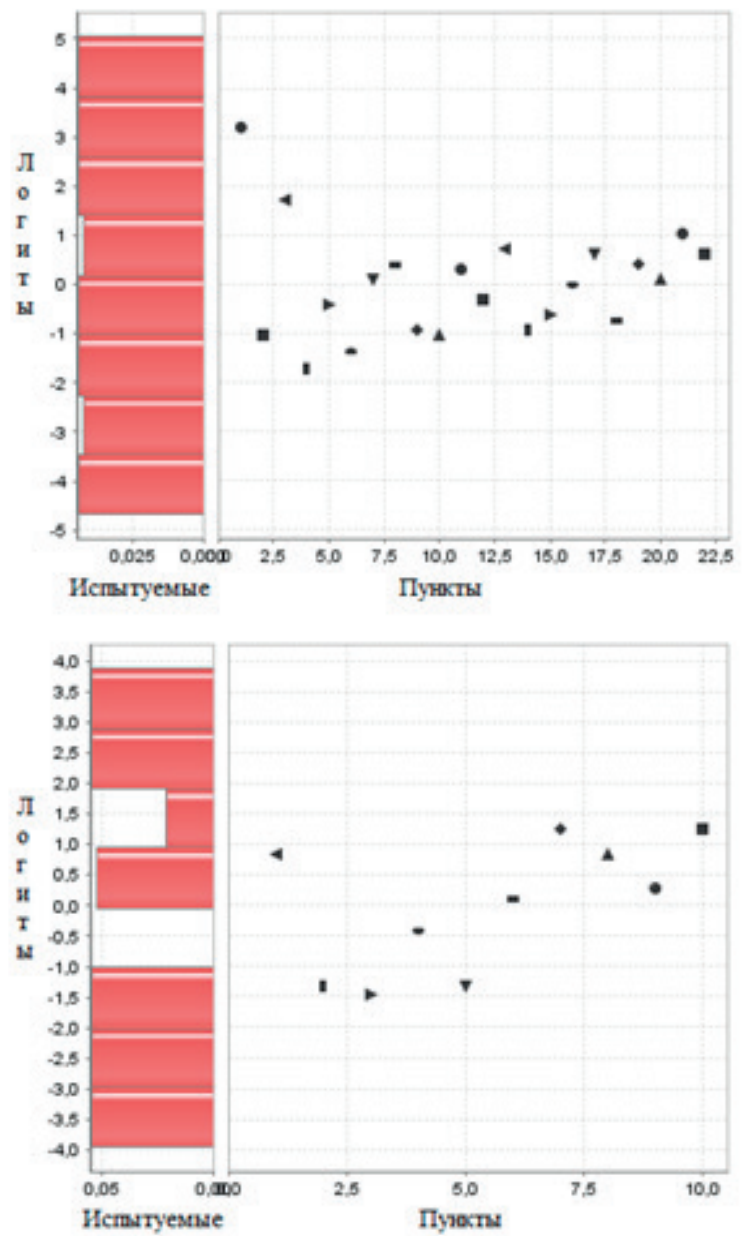

Рисунок 4. - Карта распределения мер выражсенности нарушения мышления у испытуемых и трудностей пунктов исходной и 10-пунктовой методики «Простые аналогии»
Таким образом, при улучшении одних психометрических показателей методики «Простые аналогии» (конструктная валидность) наблюдается ухудшение других показателей (количество слоев, Индекс надежности). Методика способна оценить узкий диапазон выраженности нарушения. Кроме того, не удалось получить одномерного варианта методики.

Анализ методики «Сложные аналогии» выявил схожую ситуацию. Удаление пунктов, нарушающих конструктную валидность, привело к резкому ухудшению показателя количества слоев (с 3,1 до 1,8) и Индекса надежности (с 0,82 до 0,55). Диапазон выраженности нарушения мышления, который способны оценить пункты методики, также очень узкий и одномерного варианта получить не удалось.

\section{Выводы}

1. Исходная методика «Существенные признаки» при использовании ее для оценки нарушения мышления при шизофрении не удовлетворяет психометрическим требованиям (содержит пункты, нарушающие конструктную валидность, не является одномерной).

2. Оптимальными психометрическими характеристиками обладает 9-пунктовая методика «Существенные признаки»: трудность пунктов соответствует средней степени выраженности исследуемого конструкта; методика имеет сбалансированную метрическую структуру, характеризующуюся адекватным соответствием распределения оцениваемых уровней нарушения мышления и диагностических трудностей ответных категорий пунктов; задания имеют адекватную конструктную валидность; Индекс надежности равен 0,86 ; методика способна дифференцировать 3 уровня выраженности нарушения мышления и является одномерной.

3. На всех этапах анализа методик «Простые аналогии» и «Сложные аналогии» обнаруживается несоответствие ряда показателей психометрическим требованиям, возможность использования этих методик будет определяться результатами согласованности их оценок с клиническими данными.

\section{Литература}

1. Ассанович, М. А. Клиническая психодиагностика : учеб. пособие / М. А. Ассанович. - Минск : Беларусь, 2012. - $344 \mathrm{c}$.

2. Ассанович, М. А. Статистическое обоснование критериев оценки выраженности измеряемого конструкта в клинической психодиагностике / М. А. Ассанович // Психиатрия, психотерапия и клиническая психология. - 2014. - № 2 (16). - C. 9-18.

3. Фер, Р. М. Психометрика. Введение / Р. М. Фер, В. Р. Бакарак ; пер. с англ. А. С. Науменко, А. Ю. Попова ; под ред. Н. А. Батурина, Е. В. Эйдмана. - Челябинск : Издательский центр ЮУрГУ, 2010. - 445 с.

4. Рубинштейн, С. Я. Экспериментальные методики патопсихологии. - Москва : ЗАО Изд-во ЭКСМО-Пресс, 1999. $-448 \mathrm{c}$

5. Association of formal thought disorder in schizophrenia with structural brain abnormalities in language-related cortical regions / B. Sans-Sansa [et al.] // Schizophrenia Research. - 2013. - Vol. 146 (1-3). - P. 308-313.

6. Baghaei, P. The Rasch Model as a Construct Validation Tool / P. Baghaei // Rasch Measurement Transactions. 2008. - Vol. 22. - P. 1145-1146.

7. Boone, W. J. Rasch Analysis in the Human Scienses / W. J. Boone, J. R. Staver, M. S. Yale. - New York; London : Springer, 2014. $-482 \mathrm{p}$.

8. Hamm, J. A. Disorganization and Individual Psychotherapy for Schizophrenia: A Case Report of Metacognitive Reflection and Insight Therapy / J. A. Hamm, R. L. Firmin // Journal of Contemporary Psychotherapy. - 2016. - Vol. 46 (4). - P. 227-234.

9. Maurer, K. Rasch Scaling of a Screening Instrument: Assessing Proximity to Psychosis Onset by the ERIraos 
Checklist [Electronic resource] / K. Maurer, H. Häfner // SAGE Open. - Mode of access: http://journals.sagepub. com/doi/10.1177/2158244014545326. - Date of access: 03.02.2017.

10. Paraschakis, A. Pipamperone augmentation of clozapine and sodium valproate in refractory schizophrenia: A case report / A. Paraschakis // Clinical Neuropharmacology. 2014. - Vol. 137 (2). - P. 60-61.

11. PKNOX2 is associated with formal thought disorder in schizophrenia: A meta-analysis of two genome-wide association studies / K.-S. Wang [et al.] // Journal of Molecular Neuroscience. - 2012. - Vol. 48. - P. 265-272.

12. Structural correlates of formal thought disorder in schizophrenia: An ultra-high field multivariate morphometry study / L. Palaniyappan [et al.] // Schizophrenia Research. - 2015. - Vol. 168 (1-2). - P. 305-312.

\section{References}

1. Assanovich MA. Klinicheskaja psihodiagnostika. Minsk: Belarus; 2012. 344 p. (Russian).

2. Assanovich MA. Statisticheskoe obosnovanie kriteriev ocenki vyrazhennosti izmerjaemogo konstrukta $\mathrm{v}$ klinicheskoj psihodiagnostike. Psihiatrija, psihoterapija $i$ klinicheskaja psihologija. 2014;2(16):9-18. (Russian).

3. Fer RM, Bakarak VR. Psihometrika. Vvedenie. Cheljabinsk: Izdatelskij centr JuUrGU; 2010. 445 p. (Russian).

4. Rubinshtejn SJa. Jeksperimentalnye metodiki patopsihologii. Moskva: ZAO Izd-vo JeKSMO-Press; 1999. 448 p. (Russian).
5. Sans-Sansa B, McKenna PJ, Canales-Rodríguez EJ, OrtizGil K, López-Araquistain L, Sarró S, et al. Association of formal thought disorder in schizophrenia with structural brain abnormalities in language-related cortical regions. Schizophrenia Research. 2013;146(1-3):308-313.

6. Baghaei P. The Rasch Model as a Construct Validation Tool. Rasch Measurement Transactions. 2008;22:11451146.

7. Boone WJ, Stave JR, Yale MS. Rasch Analysis in the Human Scienses. New York. London: Springer; 2014. $482 \mathrm{p}$.

8. Hamm, JA, Firmin RL. Disorganization and Individual Psychotherapy for Schizophrenia: A Case Report of Metacognitive Reflection and Insight Therapy. Journal of Contemporary Psychotherapy. 2016;46(4):227-234.

9. Maurer K, Häfner H. Rasch Scaling of a Screening Instrument: Assessing Proximity to Psychosis Onset by the ERIraos Checklist. SAGE Open. Available at: http:// journals.sagepub.com/doi/10.1177/2158244014545326. (accessed 03.02.2017).

10. Paraschakis A. Pipamperone augmentation of clozapine and sodium valproate in refractory schizophrenia: A case report. Clinical Neuropharmacology. 2014;137(2):60-61.

11. Wang K.-S, Zhang Q, Liu X, Wu L, Zeng M. PKNOX2 is associated with formal thought disorder in schizophrenia: A meta-analysis of two genome-wide association studies. Journal of Molecular Neuroscience. 2012;48:265-272.

12. Palaniyappan L, Mahmood J, Balain V, Mougin O, Gowland PA, Liddle PF. Structural correlates of formal thought disorder in schizophrenia: An ultra-high field multivariate morphometry study. Schizophrenia Research. 2015;168(1-2):305-312.

\title{
ANALYSIS OF PSYCHOMETRIC PROPERTIES OF METHODS "ESSENTIAL FEATURES", "SIMPLE ANALOGIES", "DIFFICULT ANALOGIES" USED TO ASSESS THOUGHT DISORDER IN PATIENTS WITH SCHIZOPHRENIA
}

\author{
Dziamianava L. V.
}

Educational Institution «Grodno State Medical University», Grodno, Belarus

Background. Psychometric standardization of diagnostic methods is an actual trend in modern medical psychology. The purpose of this study was to carry out the Rasch analysis of psychometric properties of the methods "Essential features", "Simple analogies", "Difficult analogies" used to assess thought disorder in patients with schizophrenia.

Material and methods. The study involved 122 patients. All the patients met criteria for schizophrenia. The difficulty of questions was considered, construct validity was estimated, reliability coefficients and discriminative index were calculated.

Results. The method "Essential features" was regenerated into 9-items, as a result an optimal combination of psychometric indices was obtained. All the stages of the analysis of the "Simple analogy" and "Difficult analogy" methods revealed a discrepancy between some indicators and psychometric requirements.

Conclusion. The possibility of using these techniques will be determined by the results of evaluation of their consistency with clinical data.

Keywords: schizophrenia, thought disorder, psychometric estimation, Rasch model 